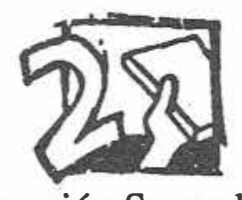

http://doi.org/10.15359/ree.2004-7.11

Educación Secundaria

\title{
EL PROGRAMA "REGLA Y COMPÁS". UNA EXPERIENCIA UTILIZANDO UN SOFTWARE GRATUITO PARA GENERAR AMBIENTES DE APRENDIZAJE EN MATEMÁTICA.
}

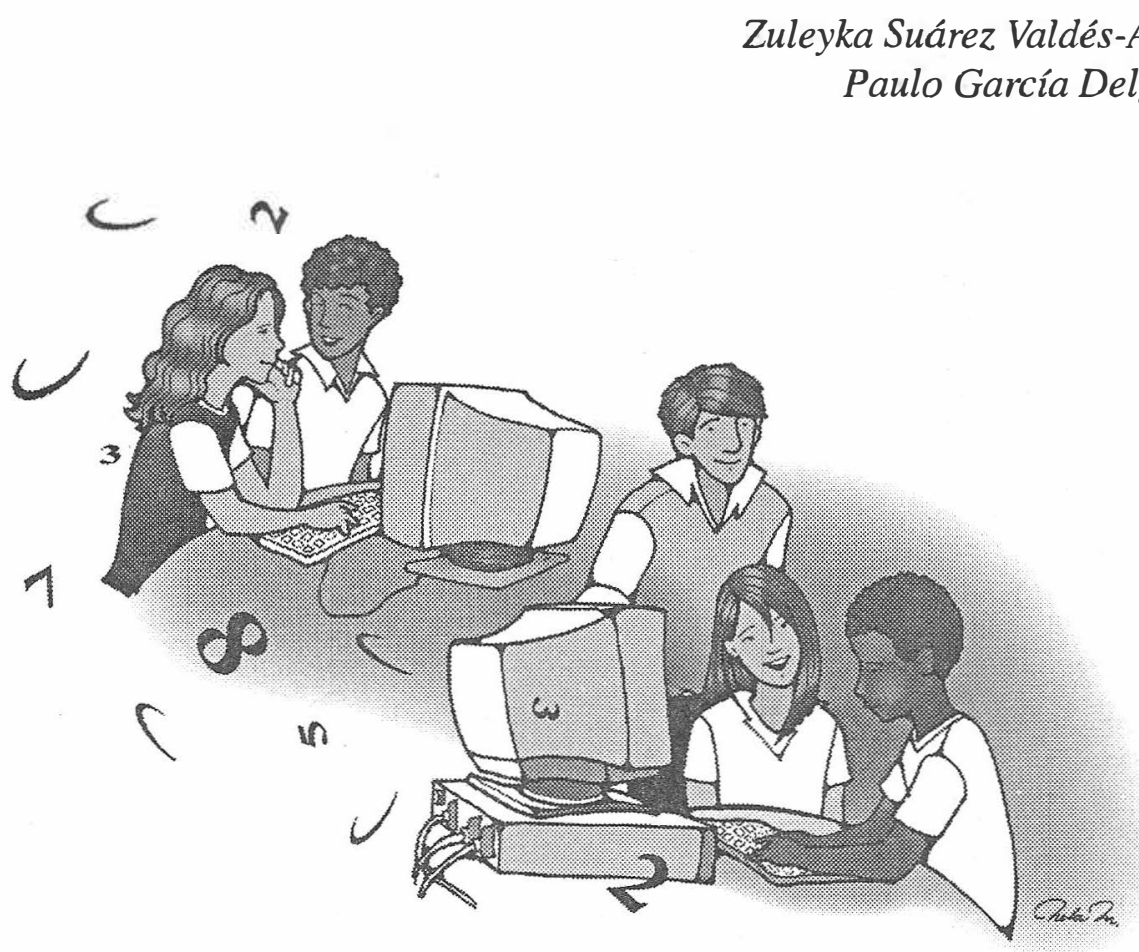

1 Licenciada en la Enseñanza de la Matemática, Universidad Nacional. Actualmente se encuentra finalizando estudios de Maestría en Educación, con énfasis en Docencia Universitaria en la Universidad Nacional. Labora como docente en el Colegio Bilingüe Santa Cecilia y en la Universidad Interamericana. Ha sido ponente en el III CIEMAC y en el I y IV Festival Internacional de Matemática.

2 Licenciado en la Enseñanza de la Matemática, Universidad Nacional. Actualmente se encuentra realizando estudios de Maestría en Matemática Educativa en la Universidad de Costa Rica. Labora como docente en el Instituto Tecnológico de Costa Rica. Ha sido ponente en el III CIEMAC y IV Festival Internacional de Matemática. 
El programa "Regla y Compás", sirve para apoyar los procesos de enseñanza y aprendizaje de la matemática en el área de geometria, mediante el uso de la computadora. Una de las ventajas de este es que puede ser adquirido de forma gratuita en Internet. Se analizará, como el uso de este tipo de programas permiten fomentar el trabajo cooperativo $e$ interdisciplinario. Para esto se abordarán las experiencias obtenidas en el desarrollo del trabajo de investigación "Enseñanza de la matemática en la educación secundaria con apoyo de software libre tomado de Internet: el caso Regla y Compás"; el cual permitió la elaboración de tresmanuales, a saber: manual del usuario, manual de las y los estudiantes y manual de el y la docente. Lo anterior con el fin de facilitar la enseñanza de la geometría por medio de 32 sesiones de práctica y aprendizaje. posiciones con respecto a temas como los valores y definir nuevos enfoques para tratar las actitudes, habilidades y contenidos necesarios para que los y las estudiantes se integren a un mundo que evoluciona rápidamente y los obliga a actuar de forma crítica, conscientes de su propia cultura y a analizar el contexto social del cual forman parte para que logren responder a los retos que surgen en su diario vivir.

En el caso particular de la enseñanza de la matemática, en uno de sus fines se plantea que los y las estudiantes puedan explorar, predecir e incluso cometer errores para ganar confianza en su propia capacidad de resolver problemas. Con el programa "Regla y Compás" se puede alcanzar este fin de una manera sencilla, pues es fácil de aprender a utilizar y permite estudiar diferentes temas contemplados dentro de los programas de estudio vigentes del $\mathrm{Mi}$ nisterio de Educación Pública.

\section{Importancia del trabajo}

Existe una dificultad con diversos programas computacionales que apoyan los procesos de enseñanza y aprendizaje de la matemática, pues pese a su gran calidad y potencial presentan una limitación: requieren licencias, las cuales implican un gasto que pocos colegios de la educación pública pueden costear. Esto podría resolverse de dos maneras: la primera es diseñar y programar el material computacional, lo cual debido a la escasa formación en programación y el tiempo que se requiere hace que los y las docentes no lo tomen en cuenta; la segunda 
The program "Regla y Comp $\ddagger s \neg$ ("Ruler and Compasses") is useful to support the teaching and learning of Math processes in geometry by means of a computer. One of the advantages it offers is that it may be obtained gratuitously in the Internet. The way in which this type of programs allows for cooperative and interdisciplinary work will be analyzed. For this purpose, the experiences obtained during the research project "Teaching high school mathematics with free online software: the Ruler and Compasses case" will be discussed. The latter enabled the elaboration of three manuals: user's manual, student's manual and adolescent's manual. The objective is to facilitate the teaching of Geometry through 32 practice and learning sessions. opción es "bajar" programas computacionales gratuitos en Internet que a pesar de estar diseñados por expertos no suelen estar acompañados de orientaciones didácticas para su empleo. Esta última opción es la que se adopta mediante el programa "Regla y Compás”; el cual se exploró desde el punto de vista de las necesidades de la educación costarricense, específicamente en el área de geometría. Como resultado de esto se diseñaron 32 sesiones de aprendizaje y práctica que permiten a los y las estudiantes trabajar de manera diferente y amena los distintos contenidos.

\section{Consideraciones teóricas}

El trabajo con programas computacionales permite desarrollar aspectos, tales como el trabajo cooperativo, el cual se aborda a continuación:

\section{Trabajo cooperativo}

La cooperación entre estudiantes implica que se deben desarrollar habilidades sociales y ser capaces de compartir con los demás. Lo anterior no significa que deba anularse la individualidad de cada uno de ellos y ellas, pues debemos estimular que defiendan sus propios criterios.

Algunas ventajas, de acuerdo con Paniego y Llopis (1995) citado por Yus (1995) son:

1. Mayor coordinación: Favorece la comunicación y la eficacia del trabajo.

2. Valoración positiva de los demás: Propicia un mayor entendimiento y ayuda mutua, así como la aceptación de otras ideas.

3. Mayor cohesión grupal: Se desarrolla más confianza y amistad entre los miembros del grupo.

Es importante que al formar los grupos, el y la docente tengan en cuenta la heterogeneidad en cuanto a etnias, sexos y niveles de razonamiento, así como 
que estos varíen para permitir un mayor contacto interpersonal y evitar "encasillamientos".

El profesor y profesora deben intervenir en el desarrollo del trabajo grupal, solo cuando sea necesario; su función debe ser de facilitador o facilitadora, nunca de solucionador o solucionadora de la labor planteada.

El éxito del trabajo en el laboratorio se logrará si se conoce el papel de la computadora en la educación y cómo se puede emplear esta adecuadamente. Seguidamente se aborda este aspecto:

\section{La computadora en el proceso educativo}

La computadora es una herramienta que puede ser utilizada en muchas áreas del conocimiento. Tiene gran capacidad, velocidad y precisión para realizar cálculos, operaciones y gráficos, entre otros aspectos.

El modelo de Jonassen (2003), explica el uso de la computadora en la educación centrándose en los siguientes aspectos:

1. El aprendizaje sobre la computadora.

2. El aprendizaje desde la computadora.

3. El aprendizaje con la computadora.

Con el software utilizado, se profundiza en el aprendizaje con la computadora desde el cual la misma es un recurso más en el proceso de aprendizaje. Esta es una herramienta que apoya tanto a los y las estudiantes como a los y las docentes en la construcción del conocimiento; sin embargo, debe quedar claro que la introducción de estas no va a cambiar el cómo enseñamos y el cómo aprenden nuestros y nuestras estudiantes si no repensamos qué y cómo queremos enseñar usándolas como apoyo en el proceso de aprendizaje.

Se busca con el uso de computadoras desarrollar habilidades metacognitivas y propiciar un aprendizaje cooperativo, logrando de esta forma que los y las estudiantes tengan una participación activa en la adquisición de su conocimiento, alcanzando un grado de autonomía y responsabilidad sobre su propio aprendizaje.

El aprendizaje con la computadora brinda la posibilidad de: integrar varios objetivos en una actividad, promover habilidades de pensamiento crítico y no la memorización de contenidos y, por último, aunque no menos importante, el hecho que varios alumnos y alumnas puedan hacer uso de la computadora a la vez, realizando trabajos en conjunto con lo cual se fomenta el trabajo cooperativo.

La tecnología no es la solución a los problemas de la enseñanza y aprendizaje de la matemática, pero sí es una herramienta importante que puede provocar 
una serie de cambios en los procesos educativos. Ante este hecho, los y las docentes debemos estar preparados para fomentar una actividad dinámica y exploratoria, donde los y las estudiantes desarrollen las capacidades de:

1. Buscar soluciones sin memorizar procedimientos.

2. Investigar modelos, en vez de memorizar fórmulas.

3. Formular conjeturas, en vez de realizar simples ejercicios de aplicación.

Meza (2000) propone una serie de estrategias didácticas para desarrollar procesos de enseñanza-aprendizaje de la matemática, usando la computadora. Entre estas se pueden mencionar:

1. Explorar para verificar: el y la docente planifican procesos donde el alumno y la alumna verifican que determinados resultados son válidos. Se requiere de un y una estudiante con capacidad de seguir instrucciones, dentro de un ambiente caracterizado por la conjetura, la búsqueda de precisión, el error como fuente de aprendizaje, la comunicación de resultados y la observación. Es importante que los y las estudiantes anoten los resultados de sus observaciones para que luego las compartan con el resto de los compañeros y compañeras.

2. Explorar para descubrir: el y la docente son facilitadores y planificadores del proceso. El y la estudiante deben llegar a sus propias conclusiones, interactuando con la computadora, observando e intercambiando opiniones con sus compañeros sin la guía directa del y de la docente a determinados resultados. Puede suceder que el y la estudiante arriben a conclusiones incorrectas o correctas, pero distintas a las que el profesor o la profesora esperaban, así que debemos estar preparados para situaciones inesperadas o novedosas. Al igual que en la estrategia anterior, el ambiente de aprendizaje está caracterizado por la observación, la conjetura, el error como fuente de aprendizaje, la búsqueda de precisión y la comunicación de resultados.

3. Jugarle la vuelta al software: el y la estudiante sin comprender algunos conceptos pueden arribar a conclusiones mediante la exploración del programa y la observación de los resultados, adquiriendo así los conceptos que necesitan para cumplir con lo que se le pide.

4. Ejercitación y práctica: los y las estudiantes se ejercitan y practican según sus propias necesidades. No es necesaria la supervisión constante del profesor o la profesora. Esta estrategia puede servir como plan remedial. 


\section{Proceso de selección del software}

Para seleccionar el programa "Regla y Compás" se procedió a la revisión de diversos softwares. La mayoría de los programas analizados, permitían tratar los temas de geometría y funciones. Se consideró importante seleccionar un programa computacional relacionado con la enseñanza y el aprendizaje de la geometría, pues esta área del conocimiento se estudia en casi todos los niveles educativos de la educación secundaria costarricense. Además, en los programas de matemática de la educación media se plantea que el estudio de la geomería combine la intuición, la experimentación y la lógica, de forma tal que a partir de construcciones se caractericen las figuras y se formulen deducciones lógicas; lo cual, por medio de este software, se logra llevar a cabo mediante actividades que cumplen lo anterior.

Otros aspectos tomados en cuenta para la elección del software fueron los siguientes:

1. Gratuidad: era imprescindible que el programa seleccionado pudiera ser bajado libremente de INTERNET y ser utilizado sin el pago de licencias.

2. Cobertura: los programas deberían estar ajustados a la temática contenida en el programa de matemática de la educación media costarricense (al menos una parte significativa del programa computacional debería cubrir temas del programa de matemática de la educación media).

3. Sencillez: se daría prioridad a los programas que, cumpliendo con las dos políticas anteriores, mostraran una curva de aprendizaje baja.

\section{Elaboración de guías y archivos}

Cuando tuvimos un conocimiento claro sobre el manejo del programa (del cual no teníamos ayuda), decidimos redactar guías dirigidas a todos los niveles educativos de la secundaria en los que se estudian temas de geometría; de forma que se aprovechara al máximo el software.

De acuerdo con nuestra propia experiencia y con fundamento en la revisión de bibliografía realizada, concluimos sobre la posibilidad de diseñar guías de dos tipos: unas para aprendizaje y otras para práctica de lo aprendido.

Como docentes en ejercicio teníamos los programas actuales del Ministerio de Educación Pública, que fueron consultados para la redacción de las guías. Se consultaron dichos temarios para la confección de estas y una de las sesiones del cuaderno de trabajo para el y la estudiante titulado "Enseñanza de la geometría en sétimo año con... Geometer's Sketchpad” elaborado por Luis Gerardo Meza. 
Cada guía indica el nivel, el tema por tratar, los archivos que utiliza y las instrucciones para su aprovechamiento. Además, para la mayoría de estas se agregó una sección opcional como complemento.

Se diseñaron nueve sesiones de aprendizaje y cuatro sesiones de práctica para sétimo año; para octavo año se diseñaron una sesión de aprendizaje y dos sesiones de práctica; para noveno año se diseñaron cuatro sesiones de aprendizaje y dos sesiones de práctica y para undécimo año se diseñaron nueve sesiones de aprendizaje y una sesión de práctica.

De manera paralela a la realización de las guías de los y las estudiantes se diseñaron los archivos que se usan en cada sesión, los cuales se hacían cuando se terminaba la redacción de la guía.

También se diseñó un conjunto de macros para facilitar algunas construcciones, por ejemplo, el ortocentro de un triángulo.

\section{Elaboración de los manuales}

Primero, se redactó el manual de los y las estudiantes en el cual se incluyeron cada una de las 32 guías, las cuales fueron ordenadas con base en lo establecido en los programas del Ministerio de Educación Pública para el 2003.

Al final del manual se añadieron unos ejercicios optativos para que los y las estudiantes exploren y logren un mayor desarrollo de las destrezas lógico-matemáticas.

Posteriormente, se elaboró el manual para el o la docente con el fin de orientar el trabajo que realizan los y las estudiantes en cada sesión. Este se realizó basado en las guías que conforman el manual del alumno y la alumna.

El manual del profesor o la profesora consta de una serie de guías, las cuales corresponden con el nivel y orden de las sesiones del manual de los y las estudiantes. En ellas se indica el tema por tratar, los conceptos que deben conocer los y las estudiantes antes de iniciar con el trabajo, los archivos que se necesitan y algunas recomendaciones y observaciones para dirigir y aprovechar al máximo la sesión de trabajo.

Finalmente, se elaboró el manual para el uso del programa "Regla y Compás" para la versión 2.83 en español.

Se usaron, para la redacción de este, las notas que durante el proceso de aprendizaje del programa fuimos tomando y la ayuda que el programa posee. Además, se accedió a cada uno de los botones del menú principal y los botones predefinidos para hacer una descripción exhaustiva de estos.

El programa se puede aprender de forma clara, mediante dicho manual, pues en este se va guiando al usuario por medio de diversas pantallas que facilitan la comprensión del software. 


\section{Validación}

La validación de las guías y los archivos se realizaron a través de nueve talleres con alumnos y alumnas y cuatro con docentes. Con cada uno de estos se iban perfeccionando cada una de las guías y de los archivos hasta obtener las versiones definitivas que se incluyeron en cada manual.

Se muestra a continuación una guía del estudiante con su archivo asociado, así como la correspondiente guía del profesor.

\section{SESIÓN PARA LOS Y LAS ESTUDIANTES}

\section{Geometría: Sétimo año}

\section{Sesión de aprendizaje No. 3}

Tema: Desigualdad triangular.

Objetivo: Construir intuitivamente el concepto de desigualdad triangular.

\section{Instrucciones:}

1- Cargue el archivo desig.triang1.car

2- Calcule las medidas de los lados del triángulo.

3- Con el botón "mover" realice los pasos 4, 6 y 8.

4- Mueva libremente el extremo del segmento long a marcado con $x$, para variar la longitud del lado a del triángulo.

5- Compare las medidas de b+c con a. ¿Cuál es mayor?

6- Mueva libremente el extremo del segmento long $\mathrm{b}$ marcado con $\mathrm{x}$, para variar la longitud del lado b del triángulo.

7- Compare las medidas de $\mathrm{a}+\mathrm{c}$ con b. ¿Cuál es mayor?

8- Mueva libremente los vértices $A$ o B, para variarla longitud del lado c del triángulo.

9- Compare las medidas de $\mathrm{a}+\mathrm{b}$ con $\mathrm{c}$. ¿Cuál es mayor?

10- Establezca una conjetura basándose en los pasos 5,7 y 9 .

\section{OPCIONAL}

1- ¿Ocurrirá lo mismo con otros triángulos?

2- Para comprobarlo cargue el archivo triángulo.car

3- En él, halle las medidas de cada lado y compruebe, usando el botón " $a+b$ " si la suma de dos lados de este triángulo siempre es mayor que el tercer lado. Lo anterior se conoce como desigualdad triangular. 


\section{Construcción asociada}

\section{desig.triang1.car.}

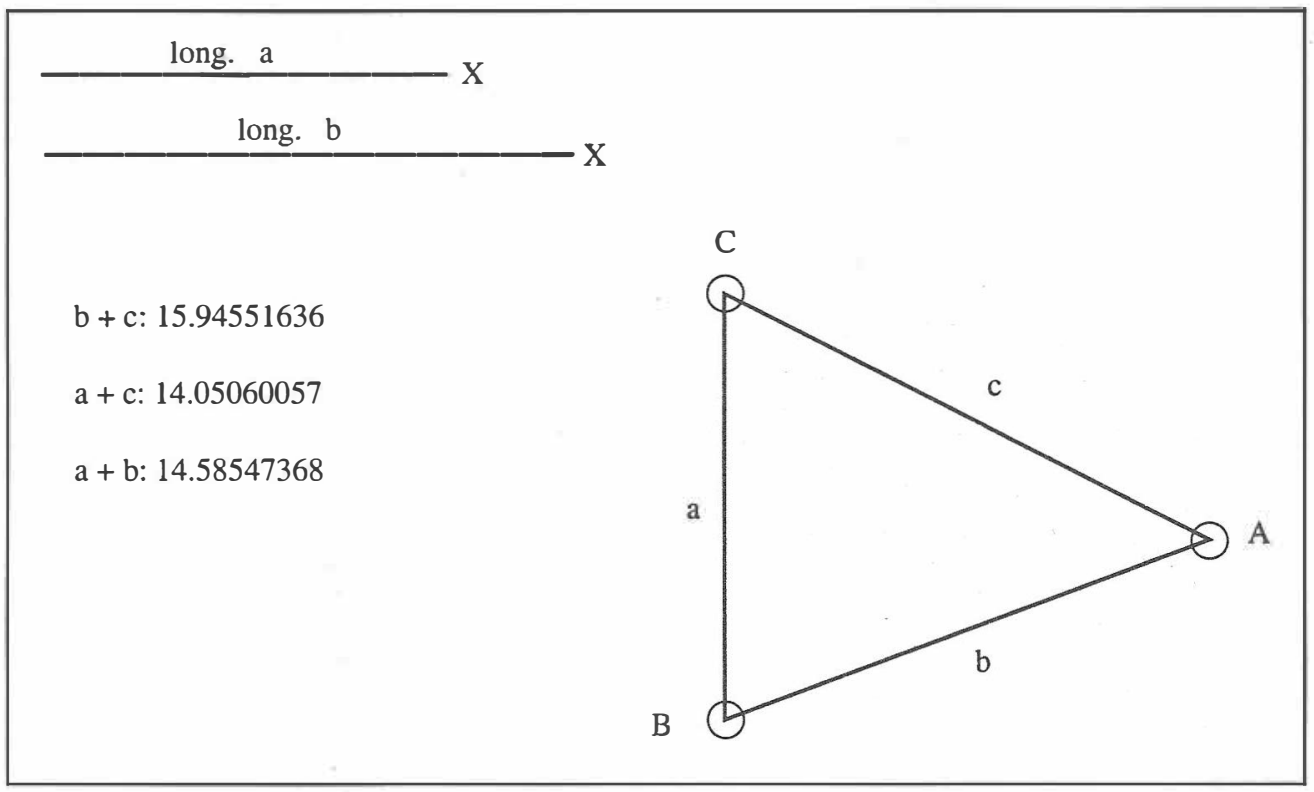

\section{triángulo.car}

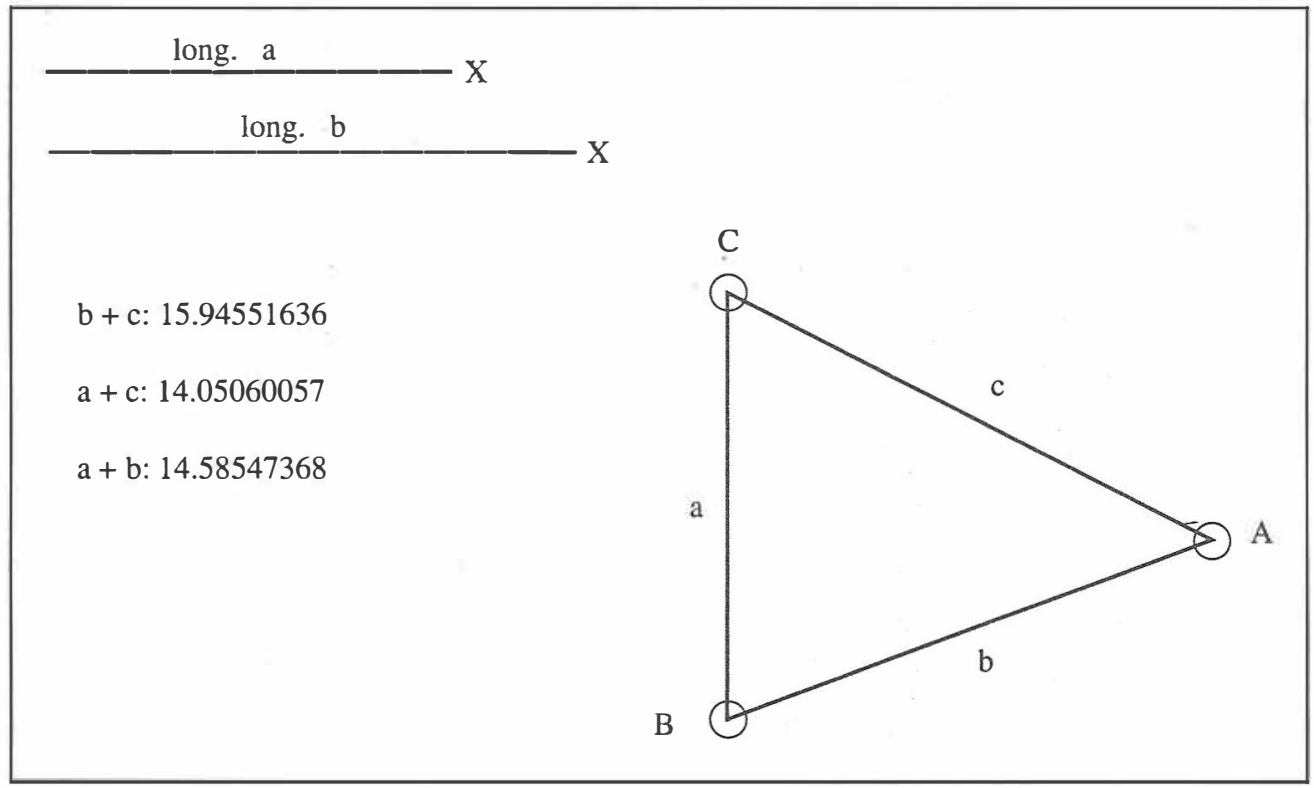




\section{GUÍA PARA EL O LA DOCENTE}

\section{Geometria: Sétimo año}

\section{Sesión de aprendizaje No. 3}

Tema: Desigualdad triangular.

Objetivo: Construir intuitivamente el concepto de desigualdad triangular.

Estrategias didácticas: Laboratorio individual o en parejas.

Nivel de dominio: Medio.

Conceptos previos: segmento, longitud, triángulo, lado de un triángulo, medida, conjetura, mayor y menor que.

Tiempo estimado: 15 minutos.

Archivo utilizado : desig.triangl.car. La sección opcional utiliza el archivo triángulo.car

\section{Recomendaciones:}

1- Asegúrese que los y las estudiantes lean el comentario inicial que aparece al cargar un archivo, pues este le brinda información sobre la construcción.

2- Para los incisos 2 y 3 de la guía y 3 de la opcional se requiere del manejo de algunos botones los cuales podrá revisar en el manual de usuario.

3- Los y las estudiantes antes de realizar esta guía en el laboratorio deben conocer el manejo de estos botones.

4- Asegúrese que en los incisos 4, 6 y 8 los y las estudiantes obtengan diversos triángulos para definir correctamente el concepto de desigualdad triangular.

5- Mediante la técnica "lluvia de ideas" se puede revisar en el aula la definición obtenida en el inciso 10, para lograr una respuesta en común.

6- El o la docente deberá prestar más atención al desarrollo de la guía en estudiantes con algún tipo de adecuación curricular o aquellos y aquellas alumnas que se les dificulte el uso de la computadora.

7- Para facilitar el aprendizaje de estudiantes con dificultades en el tema, el o la docente podrá asignarle un alumno o alumna tutora para desarrollar la guía. 
8- Si el alumno o la alumna desconfigura la construcción, deberá cargarla nuevamente. Cuando lo haga le aparecerá una pantalla para que indique si desea grabar la construcción que está cerrando, debe indicar que no.

9- El profesor o la profesora deberá tener un respaldo del archivo que se utiliza.

10- Si el y la estudiante no ejecutaron la sección opcional pueden desarrollarla como trabajo extraclase si lo desea.

\section{Observaciones:}

1- La construcción tiene un movimiento limitado por características propias del programa. Algunos alumnos y alumnas pueden notar esta limitación; debe quedarles claro que los conceptos de esta guía pueden generalizarse a más casos.

2- Con esta guía se pretende que el y la estudiante concluyan en el inciso 10, que la longitud de un lado es menor que la suma de las longitudes de los otros dos lados.

3- La sección opcional la podrán realizar aquellos y aquellas estudiantes que concluyan su trabajo antes del tiempo estimado.

4- El o la estudiante no deberá mostrar las partes ocultas de la construcción, pues puede desconfigurarla.

\section{Conclusiones}

Como un complemento en el proceso de enseñanza y aprendizaje, es posible elaborar un material didáctico, usando programas computacionales gratuitos tomados de Internet, los cuales pueden ser utilizados por parte de los y las docentes en el aula. Dichos materiales no deben ser elaborados "a la carrera", se requiere de tiempo y dedicación, además, es importante considerar una serie de aspectos tales como: la calidad del material, que logre motivar e interesar a los y las estudiantes, que se base en los objetivos del Programa de estudio vigente del MEP, que las instrucciones sean claras y que las construcciones que se diseñen sean pertinentes y no conlleven a errores.

Las construcciones elaboradas permitieron abarcar 22 de los 39 objetivos de geometría del programa de estudio del MEP en las 32 sesiones que se diseñaron, lo cual representa el $57 \%$ del total de los mismos. Lo anterior se debió a que el software no permite el trabajo con los objetivos restantes. 


\section{Referencias}

Delors, J. (1997). La educación encierra un tesoro: pautas para el siglo $X X I$. Internet. Disponible en : http://www.nuevamirada.cl/tesoro.htm

Jonassen, D. (2003). Modelos de uso de la computadora.Internet. Disponible en : http://www.guanajuato.gob.mx/seg/innova/MODEL_USO.htm

MEP. (2001). Programa de estudios de Matemática. III Ciclo. Imprenta Nacional. San José, Costa Rica.

MEP. (2001). Programa de estudios de Matemática. Educación Diversificada. Imprenta Nacional. San José, Costa Rica.

MEP (2003). Programa de Matemáticas. Internet. Disponible en :

http://www.mep.go.cr/

Meza, G.(1999). Enseñanza de la geometría en sétimo año con... Geometer's Sketchpad. Cuaderno de trabajo de la estudiante o del estudiante. Cartago, Costa Rica. Editorial Tecnológica de Costa Rica.

Meza, G. (2000). Sobre el papel de las computadoras en el proceso educativo. En Revista virtual "Matemática, educación e Internet”. Vol.1 No.1.

Meza, G. (2003). Enseñanza de la matemática complementada con estudios de computadoras. Un estudio de caso en sétimo año de un colegio público urbano. UNED, Sistema de estudios de posgrado, Doctorado en Educación.

Yus, R. (1997). Desde la cooperación en la escuela a la cooperación para el desarrollo. En Revista Educación, Desarrollo y participación democrática. Proyecto y tú... ¿Cómo lo ves?. Madrid, ACSUR-Las Segovias. Internet. Disponible en http://www.fuhem.es/CIP/EDUCA/ARTI.htm 